\title{
FHL1 and Smad4 synergistically inhibit vascular endothelial growth factor expression
}

\author{
ZHICHAO ZHOU ${ }^{1-4}$, JUMING LU ${ }^{2}$, JINGTAO DOU ${ }^{2}$, ZHAOHUI LV ${ }^{2}$, XI QIN ${ }^{3}$ and JING LIN ${ }^{4}$ \\ ${ }^{1}$ Medical School, Nankai University, Tianjin 300071; ${ }^{2}$ Department of Endocrinology, General Hospital of PLA, \\ Beijing 100853; ${ }^{3}$ Department of Medical Molecular Biology, Beijing Institute of Biotechnology, Beijing 100850; \\ ${ }^{4}$ Department of Clinical Laboratory, The First Affiliated Hospital, Chinese PLA General Hospital, Beijing 100048, P.R. China
}

Received September 17, 2012; Accepted November 15, 2012

DOI: $10.3892 / \mathrm{mmr} .2012 .1202$

\begin{abstract}
Vascular endothelial growth factor (VEGF) plays an important role in many disease states, including ischemia, chronic and acute inflammation, and pathologies associated with angiogenesis such as tumors and wounds. A number of factors regulate VEGF promoter activity and VEGF expression such as four and a half LIM domains 1 (FHL1) and Smad4. FHL1 belongs to a family of LIM-only proteins that regulate gene transcription, cell proliferation, differentiation and apoptosis. Smad4 is a tumor suppressor gene, initially identified as deleted in pancreatic carcinoma locus 4 (DPC4). The aim of this study was to determine whether FHL1 and Smad4 inhibited VEGF signaling. HepG2 cells were transfected with the VEGF-Luc reporter, Smad4 and FHL1 or Smad4 and FHL1 siRNA. Results showed that the overexpression of FHL1 and Smad4 synergistically inhibited the promoter activity, mRNA expression and secretion of VEGF, whereas knockdown of endogenous Smad4 and FHL1 had opposite effects. Moreover, the reduction of endogenous Smad4 eliminated FHL1-mediated inhibition of the VEGF promoter activity. In conclusion, a cooperative regulation of VEGF signaling by FHL1 and Smad4 was evidenced, which may provide a novel regulation mechanism underlying cancer development and progression.
\end{abstract}

\section{Introduction}

Angiogenesis, the formation of new blood vessels sprouting from the pre-existing vasculature, is pivotal for the growth

Correspondence to: Professor Jing Lin, Department of Clinical Laboratory, The First Affiliated Hospital, Chinese PLA General Hospital, 51 Fu-Cheng Lu Rd, Beijing 100048, P.R. China

E-mail: lin_jing_bj@yahoo.com.cn

Dr Xi Qin, Department of Medical Molecular Biology, Beijing Institute of Biotechnology, 27 Tai-Ping Lu Rd, Beijing 100850, P.R. China

E-mail: xiba315@126.com

Key words: co-factor, transcription factors, signal transduction of solid tumors and also plays a critical role in invasion and metastasis. The role of vascular endothelial growth factor (VEGF) in the regulation of angiogenesis has been under investigation for over a decade (1). The VEGF family includes VEGF-A, VEGF-B, VEGF-C, VEGF-D and placental growth factor (PlGF) in mammals $(2,3)$, as well as two exogenous VEGF subtypes, VEGF-E (virus genome-encoded VEGF) and VEGF-F (snake venom-derived VEGF) $(4,5)$. These VEGF molecules act through binding to several high-affinity transmembrane endothelial cell receptors, including Flt-1 (VEGFR1), KDR (VEGFR2) and Flt-4 (VEGFR3) with varying specificities. VEGF-A binds Flt-1 and KDR, while PlGF-1 and vammin (one of VEGF-Fs) selectively bind Flt-1 and KDR, respectively $(2,3,6)$. Binding of VEGF to these receptors leads to intracellular receptor phosphorylation which initiates various intracellular downstream receptor pathways, leading to endothelial cell proliferation and blood vessel formation. Tumors establish their microvasculature in part by secreting elevated amounts of VEGF. Increased levels of VEGF mRNA are found in hypoxic areas of many solid tumors $(7,8)$. Tumor suppressors, p53 (9-12), p16 (13) and the von Hippel-Lindau gene $(14,15)$ have been shown to play a role in VEGF downregulation.

In several types of cancer including bladder (16), renal cell (17), head and neck (18), colorectal $(19,20)$, cervical $(21)$, ovarian (22), lung (23) and breast cancer $(24,25)$, increased VEGF expression, either in the circulation or in tumor tissue, has been found to be correlated with decreased disease-free and shorter relapse-free intervals.

Four and a half LIM domains 1 (FHL1) belongs to a family of LIM-only proteins, containing an N-terminal half LIM domain, followed by four complete LIM domains. FHL1 regulates gene transcription, cell proliferation, differentiation and apoptosis (26). It has been reported that FHL1 inhibited VEGF promoter activity induced by HIF-1 $\alpha$ overexpression $(27,28)$ and that Smad4/deleted in pancreatic carcinoma locus 4 (DPC4) restoration affected angiogenesis, decreasing VEGF expression (29). In a previous study, we showed that FHL physically interacted with Smad4 as determined by co-immunoprecipitation (30). Furthermore, FHL1-Smad4 interaction has been shown to affect TGF- $\beta$-responsive gene transcription (30). Similarly, coexpression of FHL2 with Smad4 synergistically repressed estrogen reporter activity (31). These findings 
suggest that the interaction of FHL1 with Smad4 is required for the synergistic inhibition of VEGF signaling. The aim of this study was to determine whether FHL1 and Smad4 inhibited VEGF signaling.

\section{Materials and methods}

Plasmids. The reporter construct VEGF promoter-containing luciferase reporter (VEGF-Luc) and expression plasmids for FHL1 (32), Smad4 (33), FHL1 siRNA (32) and Smad4 siRNA (31) have been previously described.

Luciferase reporter assay. HEK293T embryonic kidney and HepG2 hepatoma cells were routinely cultured in Dulbecco's modified Eagle's medium (DMEM) (Invitrogen, Carlsbad, CA, USA) containing $10 \%$ fetal bovine serum (FBS) at $37^{\circ} \mathrm{C}$ in a humidified atmosphere of $5 \% \mathrm{CO}_{2}$ in air. Cells were transfected using Lipofectamine ${ }^{\mathrm{TM}} 2000$ (Invitrogen) with $0.2 \mu \mathrm{g}$ of VEGF-Luc reporter plasmid, $0.1 \mu \mathrm{g}$ of $\beta$-galactosidase reporter, and $1 \mu \mathrm{g}$ of expression vectors for FHL1 or Smad4, or small-interfering RNA (siRNA) vectors targeting FHL1 or Smad4. The respective empty vector was used to adjust the total amount of DNA. Luciferase and $\beta$-galactosidase activities were determined as previously described (34).

Quantitative real-time reverse transcription-polymerase chain reaction ( $q R T-P C R$ ). Total RNA was prepared using TRIzol reagent (Invitrogen) and reverse transcribed using SuperScript II Reverse Transcriptase (Invitrogen). Real-time PCR was carried out with VEGF- and GAPDH-specific primers. The sense primer for VEGF was 5'-TTCTGGGCT GTTCTCGCTTCG-3' and the antisense primer was 5'-CCC CTCTCCTCTTCCTTCTCT-3'. The sense primer for GAPDH was 5'-ACCACAGTCCATGCCATCAC-3' and the antisense primer was 5'-TCCACCACCCTGTTGCTG TA-3'. The fold change in VEGF expression was determined using the 2- $\Delta \Delta \mathrm{Ct}$ method, with GAPDH as an internal control.

Enzyme-linked immunosorbent assay (ELISA). Cells were transiently transfected with expression vectors for FHL1 or Smad4, or siRNA vectors targeting FHL1 or Smad4. At 48 h post-transfection, media were harvested for VEGF secretion assay. Human VEGF-A protein concentrations were assessed by ELISA analysis according to the manufacturer's protocol (R\&D Systems, Minneapolis, MN, USA). The values obtained were normalized to the total protein concentration in the total cell extracts prepared from each dish.

Statistical analysis. Statistical analysis was performed by using SPSS version 11.0 software. Statistical significance in the luciferase activity assays between two groups of data was determined using the unpaired t-test. Data were presented as the means $\pm \mathrm{SD}$. $\mathrm{P}<0.05$ was considered to indicate a statistically significant difference.

\section{Results}

FHL1 and Smad4 synergistically inhibit VEGF promoter activity. FHL1 has been shown to repress VEGF promoter activity in hepatocellular carcinoma cells $(27,28)$. Similar to
A

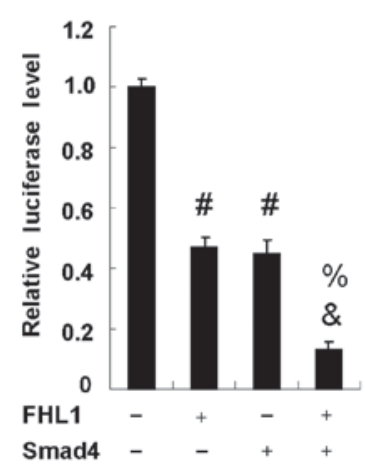

B

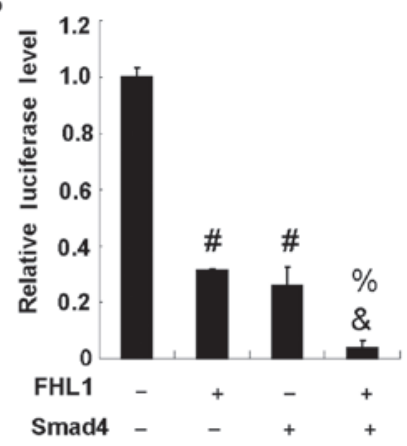

Figure 1. FHL1 and Smad4 synergistically inhibited VEGF promoter activity. (A) HepG2 or (B) HEK293T cells were cotransfected with $0.2 \mu \mathrm{g}$ of reporter VEGF-Luc, $1 \mu \mathrm{g}$ of expression plasmid for FHL1 and $1 \mu \mathrm{g}$ of plasmid expressing Smad4, as indicated. At $24 \mathrm{~h}$ post-transfection, cells were harvested and analyzed for luciferase activity. Data shown are means \pm standard deviation (SD) of triplicates of one representative experiment, repeated three times with similar results. ${ }^{*} \mathrm{P}<0.01$ vs. empty vector; ${ }^{\text {\% }} \mathrm{P}<0.01$ vs. FH1 alone; ${ }^{\circledR} \mathrm{P}<0.01$ vs. Smad4 alone.

FHL1, Smad4 has also been demonstrated to inhibit VEGF expression in pancreatic carcinoma cells. To determine whether FHL1 and Smad4 have a synergistic effect on VEGF promoter activity, hepatocellular carcinoma HepG2 cells were cotransfected with the VEGF-Luc reporter, Smad4 and FHL1. As previously reported $(27,28)$, FHL1 overexpression significantly reduced VEGF-Luc reporter activity in HepG2 cells. Similar to FHL1, Smad4 overexpression also inhibited the reporter activity. Notably, the coexpression of FHL1 with Smad4 synergistically inhibited the reporter activity due to the fact that coexpression of FHL1 with Smad4 achieved levels of inhibition greater than the sum of each individual gene expression (Fig. 1A). Similar results were observed in HEK293T cells (Fig. 1B).

Knockdown of FHL1 and Smad4 synergistically enhances $V E G F$ promoter activity. To examine the role of endogenous FHL1 and Smad4 in the repression of VEGF promoter activity, HepG2 cells were transfected with FHL1 siRNA and Smad4 siRNA. As shown in Fig. 2B, FHL1 siRNA effectively inhibited the expression of FHL1 protein at $48 \mathrm{~h}$ following transfection, whereas control siRNA had no effect. Similar results were observed when Smad4 siRNA was used. As expected, reduction of FHL1 or Smad4 enhanced the VEGF-Luc reporter activity. Similar to the results that overexpression of FHL1 and Smad4 synergistically inhibited VEGF promoter activity, knockdown of endogenous FHL1 and Smad4 synergistically enhanced VEGF promoter activity (Fig. 2A).

Abrogation of FHL1 inhibition of VEGF promoter activity by reducing endogenous Smad4. To examine the role of endogenous Smad4 in FHL1 repression of VEGF promoter activity, HepG2 cells were transfected with FHL1 or Smad4 siRNA. As shown in Fig. 3B, Smad4 siRNA effectively inhibited the expression of Smad4 protein $48 \mathrm{~h}$ after transfection, whereas control siRNA had no effect. Reduction of Smad4 enhanced the VEGF-Luc reporter activity. Notably, reduction of Smad4 eliminated FHL1 inhibition of the reporter activity, 
A

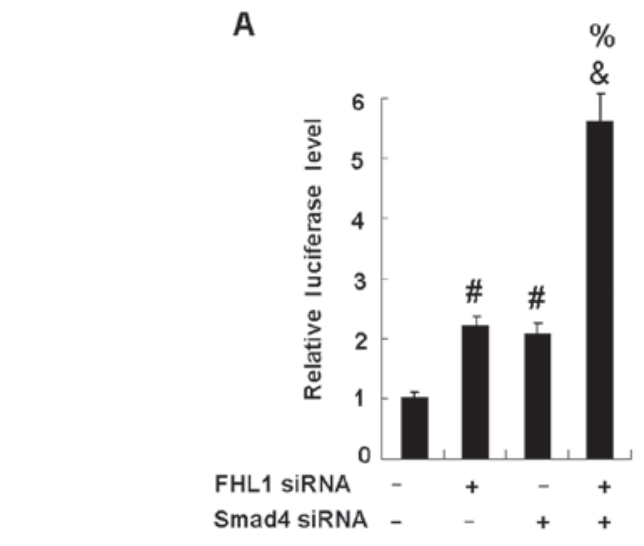

B
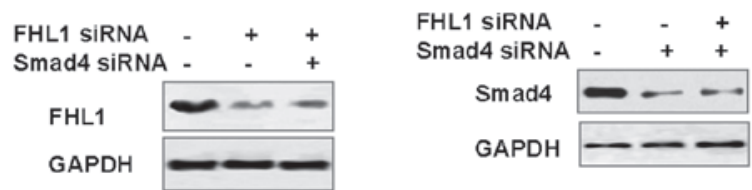

Figure 2. Knockdown of FHL1 and Smad4 synergistically enhanced VEGF promoter activity. (A) HepG2 cells were cotransfected with $0.2 \mu \mathrm{g}$ of reporter VEGF-Luc, $1 \mu \mathrm{g}$ of expression plasmid for FHL1 siRNA and $1 \mu \mathrm{g}$ of expression vector for Smad 4 siRNA, as indicated. At $48 \mathrm{~h}$ post-transfection, cells were analyzed for luciferase activity. Data shown are the means \pm standard deviation (SD) of triplicates of one representative experiment, repeated three times with similar results. ${ }^{\prime \prime} \mathrm{P}<0.01$ vs. empty vector; ${ }^{\%} \mathrm{P}<0.01$ vs. FH1 siRNA alone; ${ }^{\star} \mathrm{P}<0.01$ vs. Smad4 siRNA alone. (B) Cell lysates from (A) were used for immunoblotting with the indicated antibodies. GAPDH was used as a loading control.
A

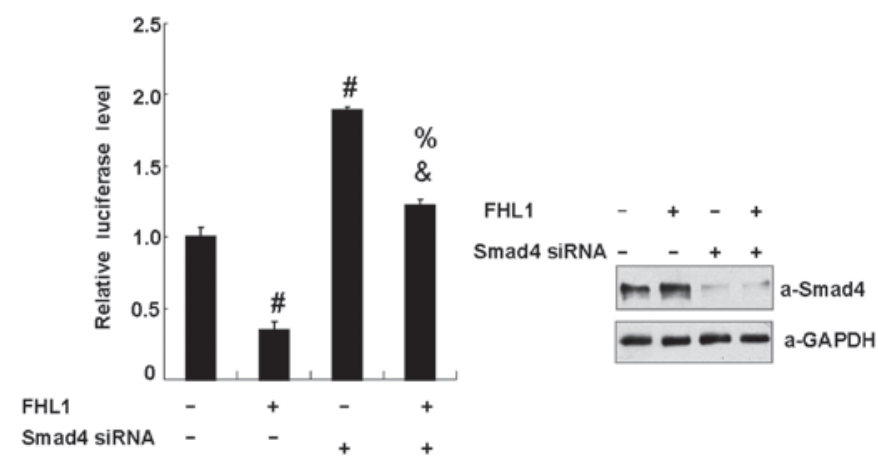

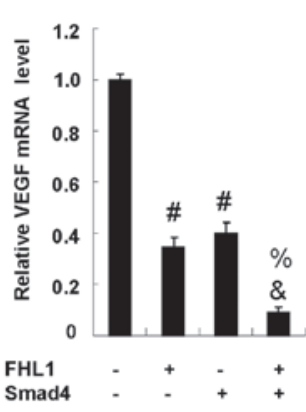

c

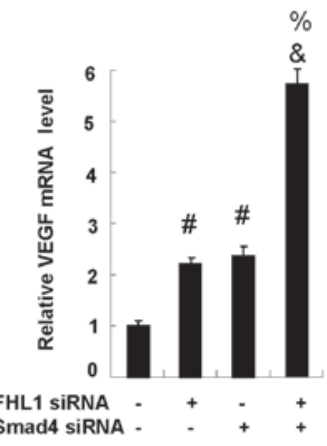

B
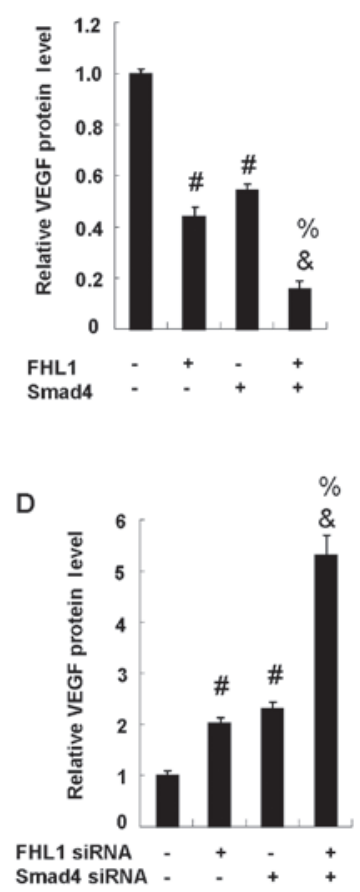

Figure 4. FHL1 and Smad4 synergistically inhibited VEGF expression. (A and B) Effects of FHL1 and Smad4 overexpression on VEGF mRNA expression and protein levels. HepG2 cells were cotransfected with FHL1 and Smad4. (A) VEGF mRNA expression was analyzed by real-time RT-PCR and (B) VEGF protein levels were determined by ELISA. (C and D) Effects of FHL1 and Smad4 siRNA on VEGF mRNA expression and protein levels. HepG2 cells were cotransfected with FHL1 and Smad4 siRNA. (C) VEGF mRNA expression was analyzed by real-time RT-PCR. (D) VEGF protein levels were determined by ELISA analysis. All the data shown are the means \pm standard deviation (SD) of triplicates of one representative experiment, repeated three times with similar results. ${ }^{\#} \mathrm{P}<0.01$ vs. empty vector; ${ }^{\%} \mathrm{P}<0.01$ vs. $\mathrm{FH} 1$ alone; ${ }^{\&} \mathrm{P}<0.01$ vs. Smad4 alone.

with FHL1, Smad4 overexpression also inhibited the mRNA expression. Notably, the coexpression of FHL1 with Smad4 synergistically inhibited the mRNA expression due to the fact that coexpression of FHL1 with Smad4 achieved levels of inhibition greater than the sum of each individual gene expression (Fig. 4A). Similar results were observed in the VEGF protein expression (Fig. 4B). As expected, the reduction of Smad4 or FHL1 enhanced VEGF expression at both mRNA (Fig. 4C) and protein levels (Fig. 4D). Of note, the reduction of FHL1 and Smad4 synergistically enhanced the mRNA and protein expression.

\section{Discussion}

In this study, we have demonstrated for the first time that FHL1 and Smad4 synergistically inhibit VEGF signaling, which has been validated by a number of in vitro experiments, including luciferase reporter, real-time reverse transcription-PCR and ELISA assays.

FHL1 was reported to play important roles in skeletal and cardiac muscle growth. FHL1 is also involved in several types of human cancer, such as breast, liver, kidney, prostate, bladder and gastric cancer $(32,35)$. Recently, FHL1 proteins have been shown to regulate the activity of transcription factors including Smad4 and HIF1 (27). FHL1 has been reported to interact with HIF1 $\alpha$, and inhibit HIF1 $\alpha$-induced VEGF promoter
FHL1 and Smad4 synergistically inhibit VEGF expression. To determine the effect of FHL1 and Smad4 on VEGF expression, HepG2 cells were cotransfected with FHL1 and Smad4, or with FHL1 siRNA and Smad4 siRNA. Overexpression of

FHL1 significantly reduced VEGF mRNA expression. As

suggesting that FHL1 represses VEGF promoter activity in a Smad4-dependent manner (Fig. 3A). 
activity and VEGF expression by blockade of HIF1 $\alpha$-HIF1 $\beta$ heterodimerization (28) and HIF-1 $\alpha$ binding to the general co-activators $\mathrm{p} 300 / \mathrm{CBP}$ (27).

Smad4, initially identified as a candidate tumor suppressor gene, is functionally inactivated at high frequency in pancreatic carcinomas and metastatic colon carcinoma $(29,36)$. Smad4 belongs to the evolutionarily conserved family of Smad proteins that are crucial for transmitting transforming growth factor- $\beta$ (TGF- $\beta$ ) superfamily signals from the cell surface to the nucleus. These proteins regulate proliferation, differentiation and cell death. Smad pathway impairment may contribute to carcinogenesis by the promotion of cell proliferation, and Smad4 inactivation in tumor patients has been closely associated with poor prognosis (37). The in vitro and in vivo experiments showed that Smad4 decreased the expression of VEGF and increased the levels of the angiogenesis inhibitor thrombospondin-1, causing human pancreatic carcinoma cells to switch from potently angiogenic to antiangiogenic (29). In a previous study, we showed that FHL1 physically and functionally interacts with Smad2, Smad3 and Smad4, and suppresses human hepatoma cell growth (30). In this study, FHL1 and Smad4 were shown to synergistically inhibit VEGF promoter reporter activity and VEGF mRNA expression and secretion levels, suggesting that FHL1-Smad4 interaction contributes to VEGF signaling-related diseases. The reduction of Smad4 eliminated the FHL1 and Smad4-mediated synergistic inhibition of the VEGF-Luc reporter activity, indicating that the inhibition of FHL1 on VEGF promoter activity occurred in a Smad4-dependent manner. However, the mechanism by which FHL1 and Smad4 synergistically control VEGF promoter activity and expression has yet to be fully elucidated. Considering that VEGF promoter harbors several potential Smad-binding sequences (29) and that FHL1 interacts with Smad4 physically and functionally, it could be concluded that FHL1 acts as a co-repressor for Smad4 in the regulation of VEGF expression.

Besides recruiting co-factors, Smad regulate transcriptional responses through physical and functional interaction with different transcription factors. Papageorgis et al (36) reported that there was physical interaction between Smad4 and HIF1 $\alpha$, which are important transcriptional factors regulating VEGF expression, providing a molecular basis for the differential regulation of target genes. Furthermore, Sánchez-Elsner et al (38) identified that HIF1 and Smad-binding motifs were within fragment -1006/-954 of the human VEGF promoter. Based on these findings, it is suggested that Smad4, HIF1 and their co-repressor FHL1 may form a complex on the promoter of VEGF and function as transcriptional co-modulators in the regulation of VEGF promoter activity and expression, and that FHL1 and Smad4 may negatively regulate HIF1 $\alpha$-induced VEGF expression synergistically.

Therefore, in this study, the cooperative transcriptional regulation of VEGF signaling by FHL1 and Smad4 was demonstrated, which might provide a novel therapeutic intervention target for VEGF signaling-related diseases.

\section{Acknowledgements}

This study was supported by the National Natural Science Foundation (81071954).

\section{References}

1. Ferrara N: VEGF and the quest for tumour angiogenesis factors. Nat Rev Cancer 2: 795-803, 2002.

2. Olsson AK, Dimberg A, Kreuger J and Claesson-Welsh L: VEGF receptor signalling - in control of vascular function. Nat Rev Mol Cell Biol 7: 359-371, 2006.

3. Ferrara N, Gerber HP and LeCouter J: The biology of VEGF and its receptors. Nat Med 9: 669-676, 2003.

4. Meyer M, Clauss M, Lepple-Wienhues A, et al: A novel vascular endothelial growth factor encoded by Orf virus, VEGF-E, mediates angiogenesis via signalling through VEGFR-2 (KDR) but not VEGFR-1 (Flt-1) receptor tyrosine kinases. EMBO J 18: 363-374, 1999.

5. Yamazaki Y and Morita T: Molecular and functional diversity of vascular endothelial growth factors. Mol Divers 10: 515-527, 2006.

6. Yamazaki Y, Takani K, Atoda H and Morita T: Snake venom vascular endothelial growth factors (VEGFs) exhibit potent activity through their specific recognition of KDR (VEGF receptor 2). J Biol Chem 278: 51985-51988, 2003.

7. Plate KH, Breier G, Weich HA and Risau W: Vascular endothelial growth factor is a potential tumour angiogenesis factor in human gliomas in vivo. Nature 359: 845-848, 1992.

8. Shweiki D, Itin A, Soffer D and Keshet E: Vascular endothelial growth factor induced by hypoxia may mediate hypoxia-initiated angiogenesis. Nature 359: 843-845, 1992.

9. Dameron KM, Volpert OV, Tainsky MA and Bouck N: Control of angiogenesis in fibroblasts by $\mathrm{p} 53$ regulation of thrombospondin-1. Science 265: 1582-1584, 1994.

10. Kieser A, Weich HA, Brandner G, Marme D and Kolch W: Mutant p53 potentiates protein kinase $\mathrm{C}$ induction of vascular endothelial growth factor expression. Oncogene 9: 963-969, 1994.

11. Mukhopadhyay D, Tsiokas L and Sukhatme VP: Wild-type p53 and v-Src exert opposing influences on human vascular endothelial growth factor gene expression. Cancer Res 55: 6161-6165, 1995.

12. Volpert OV, Dameron KM and Bouck N: Sequential development of an angiogenic phenotype by human fibroblasts progressing to tumorigenicity. Oncogene 14: 1495-1502, 1997.

13. Harada $\mathrm{H}$, Nakagawa $\mathrm{K}$, Iwata $\mathrm{S}$, et al: Restoration of wild-type p16 down-regulates vascular endothelial grow th factor expression and inhibits angiogenesis in human gliomas. Cancer Res 59: 3783-3789, 1999.

14. Siemeister G, Weindel K, Mohrs K, Barleon B, Martiny-Baron G and Marme D: Reversion of deregulated expression of vascular endothelial growth factor in human renal carcinoma cells by von Hippel-Lindau tumor suppressor protein. Cancer Res 56: 22992301, 1996.

15. Mukhopadhyay D, Knebelmann B, Cohen HT, Ananth S and Sukhatme VP: The von Hippel-Lindau tumor suppressor gene product interacts with $\mathrm{Sp} 1$ to repress vascular endothelial growth factor promoter activity. Mol Cell Biol 17: 5629-5639, 1997.

16. Inoue K, Slaton JW, Karashima T, et al: The prognostic value of angiogenesis factor expression for predicting recurrence and metastasis of bladder cancer after neoadjuvant chemotherapy and radical cystectomy. Clin Cancer Res 6: 4866-4873, 2000.

17. Jacobsen J, Rasmuson T, Grankvist K and Ljungberg B: Vascular endothelial growth factor as prognostic factor in renal cell carcinoma. J Urol 163: 343-347, 2000.

18. Eisma RJ, Spiro JD and Kreutzer DL: Vascular endothelial growth factor expression in head and neck squamous cell carcinoma. Am J Surg 174: 513-517, 1997.

19. Wong MP, Cheung N, Yuen ST, Leung SY and Chung LP: Vascular endothelial growth factor is up-regulated in the early pre-malignant stage of colorectal tumour progression. Int $\mathrm{J}$ Cancer 81: 845-850, 1999.

20. Amaya H, Tanigawa N, Lu C, et al: Association of vascular endothelial growth factor expression with tumor angiogenesis, survival and thymidine phosphorylase/platelet-derived endothelial cell growth factor expression in human colorectal cancer. Cancer Lett 119: 227-235, 1997.

21. Guidi AJ, Abu-Jawdeh G, Berse B, et al: Vascular permeability factor (vascular endothelial growth factor) expression and angiogenesis in cervical neoplasia. J Natl Cancer Inst 87: 1237-1245, 1995.

22. Boocock CA, Charnock-Jones DS, Sharkey AM, et al: Expression of vascular endothelial growth factor and its receptors flt and KDR in ovarian carcinoma. J Natl Cancer Inst 87: 506-516, 1995. 
23. O'Byrne KJ, Koukourakis MI, Giatromanolaki A, et al: Vascular endothelial growth factor, platelet-derived endothelial cell growth factor and angiogenesis in non-small-cell lung cancer. $\mathrm{Br}$ J Cancer 82: 1427-1432, 2000.

24. Brown LF, Berse B, Jackman RW, et al: Expression of vascular permeability factor (vascular endothelial growth factor) and its receptors in breast cancer. Hum Pathol 26: 86-91, 1995.

25. Yoshiji H, Gomez DE, Shibuya $M$ and Thorgeirsson UP: Expression of vascular endothelial growth factor, its receptor, and other angiogenic factors in human breast cancer. Cancer Res 56: 2013-2016, 1996.

26. Kadrmas JL and Beckerle MC: The LIM domain: from the cytoskeleton to the nucleus. Nat Rev Mol Cell Biol 5: 920-931, 2004.

27. Hubbi ME, Gilkes DM, Baek JH and Semenza GL: Fourand-a-half LIM domain proteins inhibit transactivation by hypoxia-inducible factor 1. J Biol Chem 287: 6139-6149, 2012.

28. Lin J, Qin X, Zhu Z, Mu J, et al: FHL family members suppress vascular endothelial growth factor expression through blockade of dimerization of HIF1 $\alpha$ and HIF1 $\beta$. IUBMB Life 64: 921-930, 2012.

29. Schwarte-Waldhoff I, Volpert OV, Bouck NP, et al: Smad4/ DPC4-mediated tumor suppression through suppression of angiogenesis. Proc Natl Acad Sci USA 97: 9624-9629, 2000.

30. Ding L, Wang Z, Yan J, et al: Human four-and-a-half LIM family members suppress tumor cell growth through a TGF-beta-like signaling pathway. J Clin Invest 119: 349-361, 2009.

31. Xiong Z, Ding L, Sun J, et al: Synergistic repression of estrogen receptor transcriptional activity by FHL2 and Smad4 in breast cancer cells. IUBMB Life 62: 669-676, 2010.
32. Lin J, Ding L, Jin R, et al: Four and a half LIM domains 1 (FHL1) and receptor interacting protein of $140 \mathrm{kDa}$ (RIP140) interact and cooperate in estrogen signaling. Int J Biochem Cell Biol 41: 1613-1618, 2009.

33. Sun Y, Ding L, Zhang H, et al: Potentiation of Smad-mediated transcriptional activation by the RNA-binding protein RBPMS. Nucleic Acids Res 34: 6314-6326, 2006.

34. Ding L, Yan J, Zhu J, et al: Ligand-independent activation of estrogen receptor alpha by XBP-1. Nucleic Acids Res 31: 5266-5274, 2003.

35. Matsumoto M, Kawakami K, Enokida H, et al: CpG hypermethylation of human four-and-a-half LIM domains 1 contributes to migration and invasion activity of human bladder cancer. Int J Mol Med 26: 241-247, 2010.

36. Papageorgis P, Cheng K, Ozturk S, et al: Smad4 inactivation promotes malignancy and drug resistance of colon cancer. Cancer Res 71: 998-1008, 2011.

37. Yatsuoka T, Sunamura M, Furukawa T, et al: Association of poor prognosis with loss of $12 \mathrm{q}, 17 \mathrm{p}$, and $18 \mathrm{q}$, and concordant loss of $6 \mathrm{q} / 17 \mathrm{p}$ and $12 \mathrm{q} / 18 \mathrm{q}$ in human pancreatic ductal adenocarcinoma. Am J Gastroenterol 95: 2080-2085, 2000.

38. Sánchez-Elsner T, Botella LM, Velasco B, et al: Synergistic cooperation between hypoxia and transforming growth factor-beta pathways on human vascular endothelial growth factor gene expression. J Biol Chem 276: 38527-38535, 2001 